\title{
Novel Precomputation Schemes for Elliptic Curve Cryptosystems
}

\author{
Patrick Longa and Catherine Gebotys \\ Department of Electrical and Computer Engineering, \\ University of Waterloo, Canada \\ \{plonga, cgebotys\}@uwaterloo.ca
}

\begin{abstract}
We present an innovative technique to add elliptic curve points with the form $P \pm Q$, and discuss its application to the generation of precomputed tables for the scalar multiplication. Our analysis shows that the proposed schemes offer, to the best of our knowledge, the lowest costs for precomputing points on both single and multiple scalar multiplication and for various elliptic curve forms, including the highly efficient Jacobi quartics and Edwards curves.
\end{abstract}

Keywords: Elliptic curve cryptosystem, scalar multiplication, multiple scalar multiplication, precomputation scheme, conjugate addition.

\section{Introduction}

In mid 80's, Miller and Koblitz independently proposed the use of elliptic curves for cryptographic purposes [816]. Since then, Elliptic Curve Cryptography (ECC) has gained increasing research and commercial interest. Scalar multiplication, denoted by $k P$, where $k$ is a scalar and $P$ is a point on the elliptic curve, is the central operation of most elliptic curve cryptosystems. A plethora of methods exist in the literature to execute this operation efficiently, mainly exploiting some efficient representation of the scalar. For instance, the Non-Adjacent Form (NAF) is a standard representation with the fewest nonzero terms using digits from the set $\{-1,0,1\}$.

In some settings, however, it is required to compute a multiple scalar multiplication with the form $k P+l Q$, where $k$ and $l$ are scalars and $P$ and $Q$ are points on the curve. In this scenario, well-known methods are Interleaving [17] and the Joint Sparse Form (JSF) [20].

A practical strategy that reduces further the number of required additions at the expense of some extra memory is the use of precomputations. In this case, a table of points is built and stored in advance (precomputation stage) for later use during the execution of the scalar multiplication itself (evaluation stage). Although these window-based methods effectively reduce the number of nonzero terms in most representations, a potential drawback is the cost of computing such a table, which grows with the window size.

Thus, it is an important research effort to minimize the cost of the precomputation stage to reduce the total cost of scalar multiplication. Further, although

M. Abdalla et al. (Eds.): ACNS 2009, LNCS 5536, pp. 71-88 2009.

(C) Springer-Verlag Berlin Heidelberg 2009 
improved elliptic curve shapes with faster explicit formulae are currently the focus of intense research [16], there is still a lack of analysis of precomputation schemes that are efficient for these settings.

In that direction, this work proposes efficient precomputation schemes and analyzes their performance on three relevant elliptic curve settings: standard elliptic curves using Jacobian coordinates, Jacobi quartics using extended coordinates [6] and Edwards curves using inverted Edwards coordinates [2].

The proposed schemes are based on the following simple idea: if $P+Q$ has been computed for two distinct points $P, Q$, the subtraction of those points only requires a few additional field operations 1 . In the remainder, we will refer to this operation, namely $P-Q(=P+(-Q))$, as "conjugate" addition. It will turn out that this operation will allow computing precomputed tables very efficiently. We apply the strategy of the conjugate addition to calculate tables of the form $d_{i} P$ and $c_{i} P \pm d_{i} Q$, which are commonly found in most single and multiple scalar multiplication algorithms.

Further, our precomputation schemes are compared and analyzed for three possible cases, which are determined by the system used to represent points: projective coordinates, affine coordinates with restriction to one inversion, and affine coordinates (without restriction in the number of inversions). Our extensive analysis allows determining which case is the most efficient for a particular scenario and for determined $I / M$ (field inversion/multiplication) ratios.

Our work is organized as follows. In Section 2, we detail some background about ECC over prime fields. Then, in Section 3 we describe our strategy to derive low-cost formulas for the conjugate addition in the different settings under study. In Section 4, we introduce the new schemes for precomputing points for tables with the forms $d_{i} P$ and $c_{i} P \pm d_{i} Q$, and discuss their costs. In Section 5 , we analyze and compare the performance of the proposed schemes with the previously most efficient methods. A discussion of some other applications of the strategy of the conjugate addition follows in Section 6. Some conclusions summarizing the contributions of this work are presented at the end.

\section{Preliminaries}

An elliptic curve $E$ over a prime field $\mathbb{F}_{p}$ is defined by the short Weierstrass equation $E: y^{2}=x^{3}+a x+b$, where $a, b \in \mathbb{F}_{p}$ and $\triangle=4 a^{3}+27 b^{2} \neq 0$, and which will be referred in the remainder as the standard elliptic curve form. The points on the curve $E$ and the identity element $\mathcal{O}$, known as the point at infinity, form an abelian group whose group law essentially consists of two basic operations: doubling $(2 P)$ and addition $(P+Q)$ of points.

The main operations in most elliptic curve-based cryptosystems have the forms $k P$ and $k P+l Q$, known as (single) scalar multiplication and multiple scalar multiplication.

${ }^{1}$ Okeya et al. [18] showed that an inversion can be saved when computing $P \pm Q$ in affine coordinates. We expand the idea to projective coordinates for which further reductions are possible. 
Affine coordinates (referred to as $\mathcal{A}$ in the remainder) uses $(x, y)$ coordinates to represent points. However, since this system requires field inversions, it is generally expensive over prime fields. When using efficient forms for the prime $p$ (as recommended by [4), it has been observed that the cost of inversion can be as high as $1 I>30 M$. For example, benchmarks by [10] and [3] show $I / M$ ratios between 30-40 and 50-100, respectively.

In efficient implementations, point representations with the form $(X: Y: Z)$, known as projective coordinates, were introduced to replace inversions. For example, an efficient case of this projective representation is given by Jacobian coordinates (referred to as $\mathcal{J}$ ), where each projective point $\left(X_{i}: Y_{i}: Z_{i}\right)$ corresponds to the affine point $\left(X_{i} / Z_{i}^{2}, Y_{i} / Z_{i}^{3}\right)$. In this case, equation $E$ acquires the form $Y^{2}=X^{3}+a X Z^{4}+b Z^{6}$, and the negative of an element $P=\left(X_{i}, Y_{i}, Z_{i}\right)$ is given by $-P=\left(X_{i},-Y_{i}, Z_{i}\right)$.

In recent years, other curve forms with faster group operations have appeared in the literature. In this work, we focus on two of them: Jacobi quartics and Edwards curves, whose explicit formulas have been found to be particularly fast. We briefly describe both curve shapes in the following. Note that we consider that constant curve parameters are fixed to small values so that the cost of performing any operation with them is negligible.

Jacobi quartic. It is defined by the curve $y^{2}=x^{4}+2 a x^{2}+1$, where $a \in \mathbb{F}_{p}$ and $a^{2} \neq 1$. The projective curve is $Y^{2}=X^{4}+2 a X^{2} Z^{2}+Z^{4}$, where a given projective point $\left(X_{i}: Y_{i}: Z_{i}\right)$ corresponds to the affine point $\left(X_{i} / Z_{i}, Y_{i} / Z_{i}^{2}\right)$. In this case, the negative of an element $P=\left(X_{i}, Y_{i}, Z_{i}\right)$ is represented by $-P=\left(-X_{i}, Y_{i}, Z_{i}\right)$. The most efficient formulae for these curves have been developed by Hisil et al. [6]7] using an extended coordinate system of the form $\left(X_{i}: Y_{i}: Z_{i}: X_{i}^{2}: Z_{i}^{2}\right)$ that will be referred to as $\mathcal{J} \mathcal{Q}$.

Edwards curve. It is defined by the curve $x^{2}+y^{2}=1+d x^{2} y^{2}$, where $d \notin\{0,1\}$. In [1], Bernstein and Lange presented explicit formulas for point operations on this curve using standard projective coordinates. Later in [2], the same authors introduced a more efficient coordinate system, known as inverted Edwards coordinates (denoted by $\mathcal{I E}$ ), where each projective point $\left(X_{i}: Y_{i}: Z_{i}\right)$ corresponds to $\left(Z_{i} / X_{i}, Z_{i} / Y_{i}\right)$ in affine. In this case, the curve equation is given by $\left(X^{2}+Y^{2}\right) Z^{2}=X^{2} Y^{2}+d Z^{4}$, where $X Y Z \neq 0$, and the negative of a point $P=\left(X_{i}, Y_{i}, Z_{i}\right)$ is given by $-P=\left(-X_{i}, Y_{i}, Z_{i}\right)$.

In Table1 we summarize the costs of the most efficient formulas in projective coordinates for the three curve forms under consideration. For complete details about formulas using $\mathcal{J}$ coordinates the reader is referred to [1112. Following the common practice in the literature, costs are expressed by the number of field multiplications $(M)$ and squarings $(S)$ that are required to perform certain operation, neglecting cheaper operations as field addition/subtraction $(A)$ and multiplication/division by small constants. Table 1 includes efficient operations using mixed coordinates, which are useful if input point(s) are represented in affine $(\mathcal{A})$ coordinates but the result is required in some projective system $\mathcal{P}$. Also, note that we have included efficient formulas exploiting pre-stored values. 
Table 1. Cost of elliptic curve point operations in projective coordinates using Jacobian $(\mathcal{J})$, inverted Edwards $(\mathcal{I} \mathcal{E})$ and extended Jacobi quartic $(\mathcal{J} \mathcal{Q})$ coordinates

\begin{tabular}{|c|c|c|c|}
\hline \multirow{2}{*}{ Point Operation } & \multicolumn{3}{|c|}{ Cost } \\
\hline & Jacobian $(\mathcal{J} ; a=-3)$ & $\operatorname{InvEdw~}(\mathcal{I E})$ & JQuartic $(\mathcal{J} \mathcal{Q})$ \\
\hline Doubling $(\mathrm{D}), 2 \mathcal{P} \rightarrow \mathcal{P}$ & $3 M+5 S$ & $3 M+4 S$ & $2 M+5 S$ \\
\hline Mixed doubling $(\mathrm{mD}), 2 \mathcal{A} \rightarrow \mathcal{P}$ & $1 M+5 S$ & $3 M+3 S$ & $7 S$ \\
\hline Tripling $(\mathrm{T}), 3 \mathcal{P} \rightarrow \mathcal{P}$ & $7 M+7 S$ & $9 M+4 S$ & $8 M+4 S$ \\
\hline Mixed tripling $(\mathrm{mT}), 3 \mathcal{A} \rightarrow \mathcal{P}$ & $5 M+7 S$ & $7 M+3 S$ & $5 M+6 S$ \\
\hline $\operatorname{Addition}^{(1)}(\mathrm{A}), \mathcal{P}+\mathcal{P} \rightarrow \mathcal{P}$ & $10 M+4 S / 9 M+3 S$ & - & $7 M+3 S$ \\
\hline Addition $($ A), $\mathcal{P}+\mathcal{P} \rightarrow \mathcal{P}$ & $11 M+5 S$ & $9 M+1 S$ & $7 M+4 S$ \\
\hline Mixed addition $(\mathrm{mA}), \mathcal{P}+\mathcal{A} \rightarrow \mathcal{A}$ & $7 M+4 S$ & $8 M+1 S$ & $6 M+3 S$ \\
\hline Mixed addition $(\mathrm{mmA}), \mathcal{A}+\mathcal{A} \rightarrow \mathcal{A}$ & $4 M+2 S$ & $7 M$ & $4 M+3 S$ \\
\hline DA with stored values, $2 \mathcal{P}+\mathcal{P} \rightarrow \mathcal{P}$ & $13 M+8 S$ & - & - \\
\hline $\mathrm{DA}, 2 \mathcal{P}+\mathcal{P} \rightarrow \mathcal{P}$ & $14 M+9 S$ & - & - \\
\hline Mixed DA $(\mathrm{mDA}), 2 \mathcal{P}+\mathcal{A} \rightarrow \mathcal{P}$ & $11 M+7 S$ & - & - \\
\hline
\end{tabular}

$\mathcal{P}$ : projective coordinates $(\mathcal{J}, \mathcal{I} \mathcal{E}$ or $\mathcal{J} \mathcal{Q}$ coordinates $)$

(1) Addition with stored values.

If, for instance, values $Z_{1}^{2}, Z_{1}^{3}, Z_{2}^{2}$ and $Z_{2}^{3}$ are available when computing a general addition in $\mathcal{J}$ coordinates then we can saved up to $2 M+2 S$. Similarly, in the case of Jacobi quartics it is possible to reduce the original cost of $7 M+4 S$ of the addition formula to $7 M+3 S$ by noting that $\left(X_{i}+Z_{i}\right)^{2}$ can be precomputed (see 6] for more details).

Finally, Table 1 also includes the highly efficient doubling-addition operation (DA) developed by Longa and Miri in [13, which involves the recurrent operation $2 P+Q$ and is more efficient than performing a traditional doubling followed by an addition using $\mathcal{J}$.

\section{Our Strategy: Conjugate Addition}

Our strategy to yield efficient precomputation schemes is based on the similarities between adding and subtracting two points. Basically, if the addition $P+Q$ takes place, then it is expected that, when subtracting the same points (i.e., $P-Q)$, most of the intermediate field operations are identical simply because $P-Q=P+(-Q)$ and the negative of a point only involves the change of at most one of the coordinate values in the point representation, as described in the previous section.

Let us illustrate the latter with the point addition formula using $\mathcal{J}$. Let $P=$ $\left(X_{1}, Y_{1}, Z_{1}\right)$ and $Q=\left(X_{2}, Y_{2}, Z_{2}\right)$ be two points on an elliptic curve $E$. If the addition $P+Q=\left(X_{3}, Y_{3}, Z_{3}\right)$ is performed using [12, formula (15)] as follows:

$$
X_{3}=\alpha^{2}-\left(4 \beta^{3}+8 Z_{2}^{2} X_{1} \beta^{2}\right), Y_{3}=\alpha\left(Z_{2}^{2} X_{1} \beta^{2}-X_{3}\right)-Z_{2}^{3} Y_{1} \beta^{3}, Z_{3}=\theta \beta
$$

where $\alpha=2\left(Z_{1}^{3} Y_{2}-Z_{2}^{3} Y_{1}\right), \beta=Z_{1}^{2} X_{2}-Z_{2}^{2} X_{1}$ and $\theta=\left(Z_{1}+Z_{2}\right)^{2}-Z_{1}^{2}-Z_{2}^{2}$, then $P-Q$ can be computed as $P+(-Q)=\left(X_{1}, Y_{1}, Z_{1}\right)+\left(X_{2},-Y_{2}, Z_{2}\right)=\left(X_{4}, Y_{4}, Z_{4}\right)$ reusing the partial values $\left(4 \beta^{3}+8 Z_{2}^{2} X_{1} \beta^{2}\right), Z_{2}^{2} X_{1} \beta^{2},-Z_{2}^{3} Y_{1} \beta^{3}, Z_{3}, Z_{1}^{3} Y_{2}$ and 
$Z_{2}^{3} Y_{1}$. The latter can be performed with the following formula for the conjugate addition:

$$
X_{4}=\gamma^{2}-\left(4 \beta^{3}+8 Z_{2}^{2} X_{1} \beta^{2}\right), Y_{4}=\gamma\left(Z_{2}^{2} X_{1} \beta^{2}-X_{4}\right)-Z_{2}^{3} Y_{1} \beta^{3}, Z_{4}=Z_{3}
$$

where $\gamma=-2\left(Z_{1}^{3} Y_{2}+Z_{2}^{3} Y_{1}\right)$. Note that the cost of the conjugate addition (2) using $\mathcal{J}$ is only $1 M+1 S$, which is significantly less than the cost of a general addition (1) (i.e., $11 M+5 S$ ). If we also consider other usually neglected operations, then the cost drops from $11 M+5 S+9 A+2(\times 2)+1(\times 4)$ to only $1 M+1 S+4 A+1(\times 2)$.

It may seem that performing this conjugate operation would involve several extra registers to store partial values temporarily. However, memory requirements can be minimized by performing $P+Q$ and $P-Q$ concurrently. For instance, a possible 35-step execution sequence for computing $P \pm Q$ using formulas (1) and (2) would be as the one shown in Table 2.

The execution of the addition/conjugate addition pair shown in Table 2 requires 8 registers only (including temporary registers and registers storing input coordinates). It is easy to verify that the memory requirement is the same as that of the addition formula alone. Thus, executing the conjugate addition does not increase the memory requirements in this case.

We have derived the conjugate addition formulas in projective coordinates (i.e., $\mathcal{J}, \mathcal{J} \mathcal{Q}$ and $\mathcal{I} \mathcal{E}$ coord.), and also in affine for the three curves of interest. The costs of these new formulas are summarized in Table 3 . We have also included the

Table 2. Pseudocode of an "interlaced" execution of an addition/conjugate addition pair in $\mathcal{J}$ coordinates

\begin{tabular}{|c|c|c|}
\hline $\begin{array}{l}\text { INPUT: } T_{1} \leftarrow X_{1}, T_{2} \leftarrow Y_{1}, T_{3} \leftarrow Z \\
\text { OUTPUT: } T_{1} \leftarrow X_{3}, T_{2} \leftarrow Y_{3}, T_{3} \leftarrow\end{array}$ & $\begin{array}{l}4 \leftarrow X_{2}, T_{5} \leftarrow Y_{2}, T \\
, T_{4} \leftarrow X_{4}, T_{5} \leftarrow Y_{4}\end{array}$ & \\
\hline $\begin{array}{lll}1 . & T_{7}=T_{3}^{2} & \left\{Z_{1}^{2}\right\}\end{array}$ & 19. $T_{4}=T_{4}+T_{7}$ & $\overline{\left\{\beta^{3}+2 Z_{2}^{2} X_{1} \beta^{2}\right\}}$ \\
\hline 2. $T_{4}=T_{4} \times T_{7}\left\{Z_{1}^{2} X_{2}\right\}$ & 20. $T_{4}=4 T_{4}$ & $\left\{4 \beta^{3}+8 Z_{2}^{2} X_{1} \beta^{2}\right\}$ \\
\hline 3. $T_{8}=T_{3} \times T_{7}\left\{Z_{1}^{3}\right\}$ & 21. $T_{6}=T_{5}-T_{2}$ & $\left\{Z_{1}^{3} Y_{2}-Z_{2}^{3} Y_{1}\right\}$ \\
\hline 4. $T_{5}=T_{5} \times T_{8}\left\{Z_{1}^{3} Y_{2}\right\}$ & 22. $T_{6}=2 T_{6}$ & $\{\alpha\}$ \\
\hline 5. $T_{8}=T_{6}^{2} \quad\left\{Z_{2}^{2}\right\}$ & 23. $T_{5}=-T_{5}-1$ & $\left\{-\left(Z_{1}^{3} Y_{2}+Z_{2}^{3} Y_{1}\right)\right\}$ \\
\hline 6. $T_{7}=T_{7}+T_{8}\left\{Z_{1}^{2}+Z_{2}^{2}\right\}$ & 24. $T_{5}=2 T_{5}$ & $\{\gamma\}$ \\
\hline 7. $T_{3}=T_{3}+T_{6}\left\{Z_{1}+Z_{2}\right\}$ & 25. $\quad T_{1}=T_{6}^{2}$ & $\left\{\alpha^{2}\right\}$ \\
\hline 8. $T_{3}=T_{3}^{2} \quad\left\{\left(Z_{1}+Z_{2}\right)^{2}\right\}$ & 26. $T_{1}=T_{1}-T_{4}$ & $\left\{X_{3}\right\}$ \\
\hline 9. $T_{3}=T_{3}-T_{7}\{\theta\}$ & 27. $\quad T_{7}=T_{2} \times T_{7}$ & $\left\{Z_{2}^{3} Y_{1} \beta^{3}\right\}$ \\
\hline 10. $\quad T_{6}=T_{6} \times T_{8}\left\{Z_{2}^{3}\right\}$ & 28. $T_{2}=T_{8}-T_{1}$ & $\left\{Z_{2}^{2} X_{1} \beta^{2}-X_{3}\right\}$ \\
\hline 11. $T_{2}=T_{2} \times T_{6}\left\{Z_{2}^{3} Y_{1}\right\}$ & 29. $T_{2}=T_{2} \times T_{6}$ & $\left\{\alpha\left(Z_{2}^{2} X_{1} \beta^{2}-X_{3}\right)\right\}$ \\
\hline 12. $T_{8}=T_{1} \times T_{8}\left\{Z_{2}^{2} X_{1}\right\}$ & 30. $\quad T_{2}=T_{2}-T_{7}$ & $\left\{Y_{3}\right\}$ \\
\hline 13. $T_{7}=T_{4}-T_{8}\{\beta\}$ & 31. $T_{6}=T_{5}^{2}$ & $\left\{\gamma^{2}\right\}$ \\
\hline 14. $T_{3}=T_{3} \times T_{7}\left\{Z_{3}=Z_{4}\right\}$ & 32. $T_{4}=T_{6}-T_{4}$ & $\left\{X_{4}\right\}$ \\
\hline 15. $T_{3}=T_{7}^{2} \quad\left\{\beta^{2}\right\}$ & 33. $T_{8}=T_{8}-T_{4}$ & $\left\{Z_{2}^{2} X_{1} \beta^{2}-X_{4}\right\}$ \\
\hline 16. $T_{6}=T_{6} \times T_{7}\left\{\beta^{3}\right\}$ & 34. $T_{8}=T_{5} \times T_{8}$ & $\left\{\gamma\left(Z_{2}^{2} X_{1} \beta^{2}-X_{4}\right)\right\}$ \\
\hline 17. $T_{8}=T_{6} \times T_{8}\left\{Z_{2}^{2} X_{1} \beta^{2}\right\}$ & 35. $T_{5}=T_{8}-T_{7}$ & $\left\{Y_{4}\right\}$ \\
\hline 18. $T_{4}=2 T_{8} \quad\left\{2 Z_{2}^{2} X_{1} \beta^{2}\right\}$ & & \\
\hline
\end{tabular}


Table 3. Costs of new conjugate additions for standard, Edwards and Jacobi quartic curves using projective $(\mathcal{J}, \mathcal{I} \mathcal{E}$ and $\mathcal{J} \mathcal{Q})$ and affine coordinates

\begin{tabular}{|l|c|c|c|}
\hline \multirow{2}{*}{ Point Operation } & \multicolumn{3}{|c|}{ Cost } \\
\cline { 2 - 4 } & Standard curve & Edwards curve & Jacobi quartic \\
\hline \hline Conjugate addition (A'), $\mathcal{P}-\mathcal{P} \rightarrow \mathcal{P}$ & $1 M+1 S$ & $4 M$ & $2 M+1 S$ \\
Addition (A), $\mathcal{P}+\mathcal{P} \rightarrow \mathcal{P}$ & $11 M+5 S$ & $9 M+1 S$ & $7 M+3 S$ \\
\hline Conjug. mixed addition $\left(\mathrm{mA}^{\prime}\right), \mathcal{P}-\mathcal{A} \rightarrow \mathcal{P}$ & $1 M+1 S$ & $4 M$ & $2 M+1 S$ \\
Mixed addition $(\mathrm{mA}), \mathcal{P}+\mathcal{A} \rightarrow \mathcal{P}$ & $7 M+4 S$ & $8 M+1 S$ & $6 M+3 S$ \\
\hline Conjug. mixed addition $\left(\mathrm{mmA}^{\prime}\right), \mathcal{A}-\mathcal{A} \rightarrow \mathcal{P}$ & $1 M+1 S$ & $3 M$ & $1 M+1 S$ \\
Mixed addition (mmA), $\mathcal{A}+\mathcal{A} \rightarrow \mathcal{P}$ & $4 M+2 S$ & $8 M$ & $5 M+3 S$ \\
\hline \hline Conjugate addition $\left(\mathrm{A}^{\prime}\right), \mathcal{A}-\mathcal{A} \rightarrow \mathcal{A}$ & $2 M+1 S$ & $4 M$ & $3 M$ \\
Mixed addition $(\mathrm{A}), \mathcal{A}+\mathcal{A} \rightarrow \mathcal{A}$ & $1 I+2 M+1 S$ & $1 I+9 M+1 S$ & $1 I+7 M+4 S$ \\
\hline
\end{tabular}

$\mathcal{P}$ : projective coordinates $(\mathcal{J}, \mathcal{I} \mathcal{E}$ or $\mathcal{J} \mathcal{Q}$ coordinates $)$.

costs of the traditional addition operations that accompany the execution of our formulas. Note that, in some cases, the traditional operations have been modified slightly so that the cost of the pair addition/conjugate addition is minimized. Refer to Appendices A-C for complete details.

As it can be seen in Table 3 the new conjugate formulas introduce significant cost reductions in comparison to traditional operations (see Table 1). In the following section, we take advantage of the latter to develop low-cost precomputation schemes.

\section{New Precomputation Method for Scalar Multiplication}

In this Section, we apply the concept of conjugate addition to derive highly efficient precomputation schemes first for tables of the form $d_{i} P$ and then for tables of the form $c_{i} P \pm d_{i} P$. We consider three possible scenarios: precomputed points are left in projective coordinates (referred to as case 1), precomputed points are calculated in projective coordinates and then converted to affine using one inversion (referred to as case 2), and precomputed points are computed and left in affine (referred to as case 3).

\subsection{Precomputation Scheme for Table of the Form $d_{i} P$}

Well-known methods to compute scalar multiplication using a precomputed table with points $d_{i} P$, where $d_{i} \in\{3,5, \ldots, m\}$, are Window- $w$ NAF ( $w$ NAF) and Fractional Window- $w$ NAF (Frac- $w$ NAF), in the case of single scalar multiplication, and the Interleaving method, in the case of multiple scalar multiplication.

We propose a recursive scheme that first tries to reach a "strategic" point and then applies efficiently the conjugate addition technique described in Section 3. In the following, we define as "strategic" to those points that can be efficiently computed and from which it is possible to calculate the maximum possible number of precomputed points at the lowest cost. The steps of our scheme are detailed in the following. 
Step 1: Computation of precomputed points. This is the main body of our scheme, and is presented in Algorithm 4.1. In this step, points can be computed in projective coordinates using operations from Table 1 (case 1), or directly in $\mathcal{A}$ (case 3 ). If projective points are to be converted to $\mathcal{A}$ (case 2), then Step 2 should be executed right after.

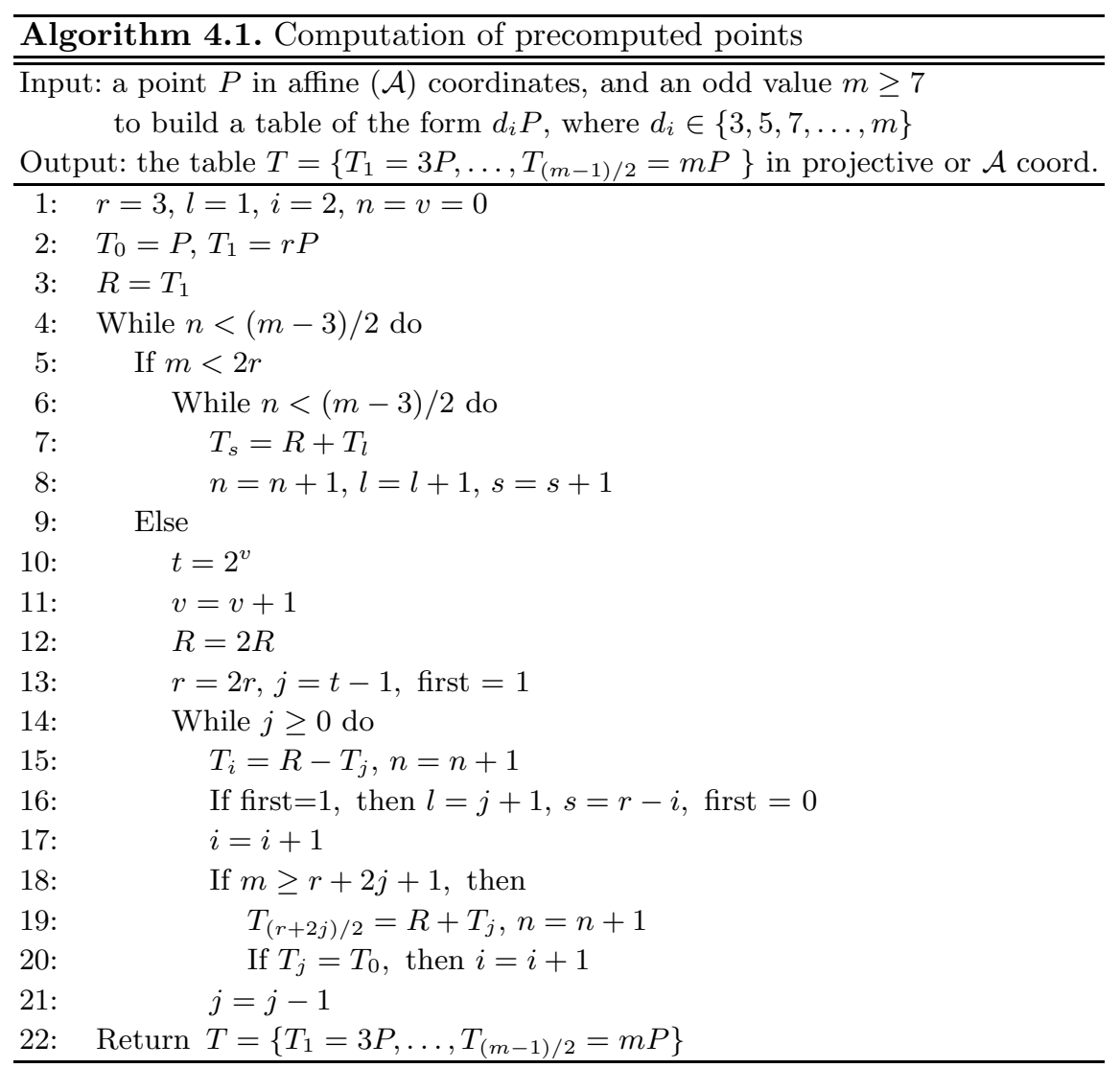

Basically, Algorithm 4.1 first reaches certain "strategic" point and then computes all the points that are close to it by efficiently performing additions and conjugate additions. The "strategic" points proposed in our scheme have the form $P_{i+1}=2 P_{i}$, for $i \in \mathbb{Z} \geq 0$ and $P_{0}=3 P$ (i.e., $6 P, 12 P, 24 P$, and so on), which are computed using a combination of one tripling (performed at the beginning, Step 2) and a sequence of doublings (Step 12). Note that there is a minimum number of close points that makes the computation of a "strategic" point worthwhile. If that minimum is not fulfilled (evaluation in Step 5) then the algorithm calculates the remaining points from the previous "strategic" point (loop beginning in Step 6). The value of such a minimum depends on the particular costs of point operations. For $\mathcal{J}, \mathcal{J} \mathcal{Q}$ and $\mathcal{I} \mathcal{E}$, we have determined that 
the lowest cost is achieved if the next "strategic" point is computed always that the $m$ value is greater or equal to such a "strategic" point (condition in Step 5).

Let us illustrate the proposed scheme with the following example.

Example 1. If $m=13$, Alg. 4.1 computes the first points as $P \rightarrow 3 P \rightarrow 6 P$, where $6 P$ is the first "strategic" point. From this, $5 P$ and $7 P$ (close points) are calculated by adding $6 P+(-P)$ and $6 P+P$. Note that the latter operation can be calculated with a conjugate addition, requiring a very low number of operations. Then, Alg. 4.1 calculates the following "strategic" point (since $m>12$ ) by doubling $6 P \rightarrow 12 P$, and finally computes close points $9 P, 11 P$ and $13 P$ by performing $12 P+(-3 P), 12 P+(-P)$ and $12 P+P$, respectively. Note again that the latter operation is also a low-cost conjugate addition.

In Appendix D, we have sketched the derivation of points for tables with different values $m$. Note that the method described does not include cases $m=3,5$. Computing the table for $m=3$ only requires one mixed tripling. For case $m=5$, $\mathcal{J} \mathcal{Q}$ and $\mathcal{J}$ coordinates, it is more efficient to compute points by performing $P \rightarrow 2 P \rightarrow 4 P$, and then obtaining $3 P$ and $5 P$ with an addition/conjugate addition pair (i.e., $4 P+(-P)$ and $4 P+P)$. For case $\mathcal{I E}$, we suggest to compute the table following the sequence $P \rightarrow 2 P \rightarrow 3 P \rightarrow 5 P$.

In the following, we describe the procedure to convert points to $\mathcal{A}$ for case 2 .

Step 2: Conversion to affine (if required). If mixed addition (or mixed DA) is significantly more efficient than general addition (or general DA) in a given setting, then it would be convenient to express the precomputed table in $\mathcal{A}$.

It is known that conversion to $\mathcal{A}$ can be achieved by calculating $\left(X_{i} / Z_{i}^{2}\right.$, $\left.Y_{i} / Z_{i}^{3}\right),\left(X_{i} / Z_{i}, Y_{i} / Z_{i}^{2}\right)$ and $\left(Z_{i} / X_{i}, Z_{i} / Y_{i}\right)$ for $\mathcal{J}, \mathcal{J} \mathcal{Q}$ and $\mathcal{I} \mathcal{E}$, respectively.

For each setting, calculation of denominators (denoted by $u_{i}$ ) can be efficiently carried out by using the well-known Montgomery' simultaneous inversion method so that the number of expensive inversions is limited to only one.

First, we compute the inverse $U=\left(u_{1} u_{2} \ldots u_{t}\right)^{-1}$, where $u_{i}$ are all distinct denominators of the expressions above from all the non-trivial points in the table $\{3 P, 5 P, \ldots, m P\}$. For $\mathcal{J}$ and $\mathcal{J} \mathcal{Q}$, the number of such denominators is reduced to only $t=(m-1) / 2-c$, where $c$ is the number of points computed via conjugate addition, since points computed with addition/conjugate addition pairs share the same coordinate $Z$ (see Appendices A-B). For $\mathcal{I} \mathcal{E}, t=m-1$ as each point has two distinct denominators, namely $X_{i}$ and $Y_{i}$.

Then, individual denominators $u_{i}$ are recovered from $U$, and the results multiplied to their corresponding numerator following the conversion expressions.

As it can be seen the use of conjugate additions reduces the cost of the Montgomery's method for the cases of $\mathcal{J}$ and $\mathcal{J} \mathcal{Q}$ coordinates. Following our explanation above, it can be easily verified that one saves $3 M+1 S$ per point computed with a conjugate addition.

Cost Analysis. The cost of the scheme proposed mainly depends on the value $m$ in the precomputed table and the curve form selected. We list in Table 4 the costs in terms of number of operations for various values $m$. As operations in $\mathcal{A}$ 
Table 4. Cost of the proposed precomputation scheme: case 1 in projective coordinates using $\mathcal{J}$ and $\mathcal{J} \mathcal{Q}$; case 2 using one inversion; and case 3 in $\mathcal{A}$

\begin{tabular}{|c|l|c|c|c|c|}
\hline \multirow{2}{*}{$m$} & \multirow{2}{*}{ Point Operation Count } & \multicolumn{2}{|c|}{ Case 1} & \multicolumn{2}{c|}{ Case 2} \\
\cline { 3 - 6 } & & $\mathcal{J}$ & $\mathcal{J Q}$ & $\mathcal{J}$ & $\mathcal{J Q}$ \\
\hline \hline 7 & $1 \mathrm{mT}+1 \mathrm{D}+1 \mathrm{~mA}+1 \mathrm{~mA}^{\prime}$ & $17 M+17 S$ & $15 M+17 S$ & $1 I+28 M+18 S$ & $1 I+24 M+20 S$ \\
9 & $1 \mathrm{mT}+1 \mathrm{D}+1 \mathrm{~mA}+1 \mathrm{~mA}^{\prime}+1 \mathrm{~A}$ & $27 M+21 S$ & $22 M+20 S$ & $1 I+43 M+22 S$ & $1 I+36 M+25 S$ \\
11 & $1 \mathrm{mT}+1 \mathrm{D}+1 \mathrm{~mA}+1 \mathrm{~mA}^{\prime}+2 \mathrm{~A}$ & $37 M+25 S$ & $29 M+23 S$ & $1 I+59 M+27 S$ & $1 I+48 M+30 S$ \\
13 & $1 \mathrm{mT}+2 \mathrm{D}+2 \mathrm{~mA}+2 \mathrm{~mA}^{\prime}+1 \mathrm{~A}$ & $39 M+31 S$ & $32 M+30 S$ & $1 I+63 M+32 S$ & $1 I+53 M+35 S$ \\
15 & $1 \mathrm{mT}+2 \mathrm{D}+2 \mathrm{~mA}+2 \mathrm{~mA}^{\prime}+1 \mathrm{~A}+1 \mathrm{~A}$ & $40 M+32 S$ & $34 M+32 S$ & $1 I+67 M+33 S$ & $1 I+57 M+37 S$ \\
\hline
\end{tabular}

\begin{tabular}{|c|l|c|}
\hline$m$ & Point Operation Count & $\begin{array}{c}\text { Case } 3 \\
\text { Standard curve }\end{array}$ \\
\hline \hline 7 & $1 \mathrm{~T}+1 \mathrm{D}+1 \mathrm{~A}+1 \mathrm{~A}^{\prime}$ & $3 I+13 M+7 S$ \\
9 & $1 \mathrm{~T}+1 \mathrm{D}+1 \mathrm{~A}+1 \mathrm{~A}^{\prime}+1 \mathrm{~A}$ & $4 I+15 M+8 S$ \\
11 & $1 \mathrm{~T}+1 \mathrm{D}+1 \mathrm{~A}+1 \mathrm{~A}^{\prime}+2 \mathrm{~A}$ & $5 I+17 M+9 S$ \\
13 & $1 \mathrm{~T}+2 \mathrm{D}+2 \mathrm{~A}+2 \mathrm{~A}^{\prime}+1 \mathrm{~A}$ & $6 I+21 M+11 S$ \\
15 & $1 \mathrm{~T}+2 \mathrm{D}+2 \mathrm{~A}+2 \mathrm{~A}^{\prime}+1 \mathrm{~A}+1 \mathrm{~A}^{\prime}$ & $6 I+23 M+12 S$ \\
\hline
\end{tabular}

coord. are relatively expensive in Jacobi quartic and Edwards curves (see Table 3), we only show the performance of case 3 in the setting of the standard curve.

Depending on the curve form selected, some additional considerations are necessary. In the case of the standard curve using $\mathcal{J}$, if the evaluation stage uses the efficient addition with two stored values, then values $Z_{i}^{2}$ and $Z_{i}^{3}$ should be computed during the precomputation stage. Naively, the latter would require $(1 M+1 S)(m-1) / 2$. However, some additional cost reductions are possible. First, the initial tripling computes the required values for point $3 P$ (i.e., $Z_{3 P}^{2}$ and $Z_{3 P}^{3}$ ) without requiring extra operations. Also, one squaring can be saved every time a doubling is performed to get any "strategic" point since values $Z_{i}^{2}$ are cached. Moreover, it is easy to see that addition and conjugate addition formulas share the same coordinate $Z$ (see Appendix A). Hence, we only require $1 M+1 S$ to get $Z_{i}^{2}$ and $Z_{i}^{3}$ for two points computed with an addition/conjugate addition pair. Finally, when performing additions using a "strategic" point $Q$, its values $Z_{Q}^{2}$ and $Z_{Q}^{3}$ are calculated in the first mixed addition, say $Q+P=$ $\left(X_{Q}, Y_{Q}, Z_{Q}\right)+\left(x_{1}, y_{1}\right)$. Thus, following general additions of the form $Q+R=$ $\left(X_{Q}, Y_{Q}, Z_{Q}\right)+\left(X_{R}, Y_{R}, Z_{R}\right)$ can be executed using an addition with four stored values, taking into account that $R$ is a point from the table and that values $Z_{R}^{2}$ and $Z_{R}^{3}$ are, hence, precalculated.

Similarly, in $\mathcal{J} \mathcal{Q}$, if the evaluation stage uses the efficient addition with the stored value $\left(X_{i}+Z_{i}\right)^{2}$, then these values should be included in the precomputation cost. We now describe a few optimizations to minimize this cost. First, one squaring can be saved every time a doubling is performed to get any "strategic" point by noting that $\left(X_{i}+Z_{i}\right)^{2}$ can be cached from a previous mixed tripling or mixed addition. Also, when performing additions with a "strategic" point $Q$, the value $\left(X_{Q}+Z_{Q}\right)^{2}$ is calculated in the first mixed addition. Then, following general additions with the same point $Q$ save one extra squaring. 
The costs including the savings described above are detailed in Table 4, case 1. For the case where points are converted to $\mathcal{A}$ (case 2), we have to also consider the cost of performing the Montgomery' simultaneous inversion method (Step $2)$. The cost of the latter in $\mathcal{J}$ and $\mathcal{J} \mathcal{Q}$ is given by $\operatorname{Cost}_{\mathcal{J} \rightarrow \mathcal{A}}=1 I+(6 L-$ 3) $M+(L) S$ and $\operatorname{Cost}_{\mathcal{J} \mathcal{Q} \rightarrow \mathcal{A}}=1 I+(5 L-3) M+(2 L) S$, respectively, where $L=(m-1) / 2$ and $m$ odd $\geq 5$. However, as described in Section 4.1, Step 2, the proposed scheme allows for some extra savings since points obtained through an addition/conjugate addition pair share the same coordinate $Z$. The reduced costs including these savings are given by

$$
\begin{gathered}
\text { Cost }_{\text {proposed } \mathcal{J} \rightarrow \mathcal{A}}=1 I+(6 L-3 c-3) M+(L-c) S \\
\text { Cost }_{\text {proposed } \mathcal{J} \mathcal{Q} \rightarrow \mathcal{A}}=1 I+(5 L-3 c-3) M+(2 L-c) S
\end{gathered}
$$

respectively, where $c$ denotes the number of points obtained using a conjugate addition. In the case of $\mathcal{I} \mathcal{E}$, the cost of the Montgomery's method is as follows

$$
\operatorname{Cost}_{\mathcal{I E} \rightarrow \mathcal{A}}=1 I+(6 L+\lceil(L-2) / L\rceil-1) M
$$

The total costs including conversion to $\mathcal{A}$ are given in Table 4 case 2. Note that in this case addition operations with stored values do not apply.

\subsection{Precomputation Scheme for Table of the Form $c_{i} P \pm d_{i} Q$}

This scenario mainly applies to methods for computing multiple scalar multiplications such as those based on JSF [20]. In this case, the application of our strategy of conjugate additions is straightforward since precomputed points have the form $c_{i} P \pm d_{i} Q$, where $c_{i}, d_{i} \in\{0,1,3,5, \ldots, m\}$, and each two points $c P \pm d P$ having $c, d \neq 0$ can be computed with an addition/conjugate addition pair.

In the following, we analyze the cost involved when precomputing points for the specific case of the efficient JSF-based algorithm by Kuang et al. 9]. Extension of the method to similar table forms easily follows.

Cost Analysis. If $P$ and $Q$ are unknown before the scalar multiplication is executed, the points $3 P, 3 Q, P \pm Q, 3 P \pm Q, P \pm 3 Q, 3 P \pm 3 Q$ required by the method by 9. need to be computed on the fly. The latter costs $2 \mathrm{mT}+2 \mathrm{mmA}+4 \mathrm{~mA}+2 \mathrm{~A}$ for case 1 (when points are left in projective coord.). With the strategy of conjugate additions, that cost reduces to $2 \mathrm{mT}+1 \mathrm{mmA}^{2}+1 \mathrm{mmA}^{\prime}+2 \mathrm{~mA}+2 \mathrm{~mA}^{\prime}+1 \mathrm{~A}+1 \mathrm{~A}^{\prime}$. Note that the advantage increases for case 2 as our approach allows saving some operations during conversion to $\mathcal{A}$, as shown in Section 4.1.

In Table 5, we show the cost performance of the proposed scheme for the considered curve shapes. Note that, in the setting of $\mathcal{J}$ and $\mathcal{J} \mathcal{Q}$, we use again the efficient addition formulas with stored values and, following the same procedure described in Section 4.1, we have minimized the impact of the computation of those partial values for case 1 . For case 2 the conversion to $\mathcal{A}$ coordinates is similar to that of the scheme from Section 4.1 and, hence, it follows the costs given by (3), (4) and (5) for $\mathcal{J}, \mathcal{J} \mathcal{Q}$ and $\mathcal{I E}$, respectively. Again, as operations in affine are relatively expensive in Edwards and Jacobi quartic curves, we only show the performance of case 3 in the setting of standard curves. 
Table 5. Cost of the proposed precomputation scheme for the $\mathrm{JSF}_{3}$ method 9]: case 1 in projective coord. using $\mathcal{J}$ and $\mathcal{J} \mathcal{Q}$; case 2 using one inversion; and case 3 in $\mathcal{A}$.

\begin{tabular}{|l|l|c|c|c|}
\hline Curve form & Point operations & Case 1 & Case 2 & Case 3 \\
\hline \hline Jacobi quartic $(\mathcal{J Q})$ & & $41 M+35 S$ & $1 I+76 M+44 S$ & - \\
Edwards $(\mathcal{I E})$ & $2 \mathrm{mT}^{\prime} 1 \mathrm{mmA}^{\prime}+1 \mathrm{mmA}^{\prime}+$ & $47 M+24 S$ & $1 I+107 M+24 S$ & - \\
Standard $(\mathcal{J})$ & $2 \mathrm{~mA}^{2} 2 \mathrm{~mA}^{\prime}+1 \mathrm{~A}+1 \mathrm{~A}^{\prime}$ & $42 M+32 S$ & $1 I+84 M+35 S$ & $6 I+30 M+16 S$ \\
\hline
\end{tabular}

\section{Performance Comparison}

In this section, we analyze and compare the proposed approach with the most efficient precomputation schemes available in the literature.

In the case of $\mathcal{J}$, Longa and Miri 13 recently proposed a highly efficient scheme, which has been shown to achieve the lowest cost among methods using only one inversion (case 2). The cost of this method (referred to as LM method in the remainder) is given by $(1 M=0.8 S)$

$$
\text { Cost }_{L M, \text { case } 2}=1 I+(9 L) M+(2 L+6) S=1 I+(10.6 L+4.8) M
$$

We now derive the cost of the LM method for case 1 using the traditional chain $P \rightarrow 2 P \rightarrow 3 P \rightarrow 5 P \rightarrow \ldots \rightarrow m P$ and the special addition due to [15], but avoiding the final conversion to $\mathcal{A}$. This involves one mixed doubling and $L$ special additions that cost $5 M+2 S$. Also, the use of additions with pre-stored values during the evaluation stage requires precalculating values $Z_{i}^{2}$ and $Z_{i}^{3}$ with a cost of $L(1 M+1 S)$. Then the total cost is

$$
\text { Cost }_{L M, \text { case } 1}=(6 L+1) M+(3 L+5) S=(8.4 L+5) M
$$

Regarding $\mathcal{I E}$ and $\mathcal{J} \mathcal{Q}$, we could not find any literature related to precomputation schemes in these settings. Hence, we analyze in the following the performance of the straightforward implementation using the traditional chain given above.

The cost of precomputation without using inversions (case 1) is given by

$$
\begin{aligned}
& \text { Cost }_{\mathcal{I} \mathcal{E}, \text { case } 1}=(9 L+2) M+(1 L+3) S=(9.8 L+4.4) M \\
& \text { Cost }_{\mathcal{J} \mathcal{Q}, \text { case } 1}=(7 L-1) M+(3 L+7) S=(9.4 L+4.6) M
\end{aligned}
$$

for $\mathcal{I} \mathcal{E}$ and $\mathcal{J} \mathcal{Q}$ coordinates, respectively. These costs are derived by adding the costs of performing one mixed doubling, one mixed addition and $(L-1)$ general additions. For case 2, the costs are given by

$$
\begin{aligned}
& \operatorname{Cost}_{\mathcal{I E}, \text { case } 2}=1 I+(15.8 L+\lceil(L-2) / L\rceil+3.4) M \\
& \operatorname{Cost}_{\mathcal{J} \mathcal{Q}, \text { case } 2}=1 I+(12 L-4) M+(5 L+7) S=1 I+(16 L+1.6) M
\end{aligned}
$$

which are derived by adding the cost of performing the Montgomery's method of simultaneous inversion to equations (8) and (9). 
Table 6. Costs of various schemes in projective (case 1) and affine (case 2); $1 M=0.8 S$

\begin{tabular}{|c|c|c|c|c|c|c|}
\hline Case & Method & Curve form & $w=3$ & $w=4$ & $w=5$ & $w=6$ \\
\hline \multirow{6}{*}{ case 1} & Proposed scheme & $\mathcal{J} \mathcal{Q}$ & $10.6 M$ & $28.6 M$ & $59.6 M$ & $116.6 M$ \\
\hline & Method 9 & $\mathcal{J Q}$ & - & $32.8 M$ & $70.4 M$ & $145.6 M$ \\
\hline & Proposed scheme & $\mathcal{I E}$ & $9.4 M$ & $28.4 M$ & $61.2 M$ & $121.6 M$ \\
\hline & Method 8 & $\mathcal{I E}$ & - & $33.8 M$ & $73.0 M$ & $151.4 M$ \\
\hline & Proposed scheme & $\mathcal{J}$ & $10.6 M$ & $30.6 M$ & $65.6 M$ & $130.6 M$ \\
\hline & LM Method (7) & $\mathcal{J}$ & - & $30.2 M$ & $63.8 M$ & $131.0 M$ \\
\hline \multirow{6}{*}{ case 2} & Proposed scheme & $\mathcal{J} \mathcal{Q}$ & - & $1 I+40.0 M$ & $1 I+86.6 M$ & $1 I+173.6 M$ \\
\hline & Method (11) & $\mathcal{J} \mathcal{Q}$ & - & $1 I+49.6 M$ & $1 I+113.6 M$ & $1 I+241.6 M$ \\
\hline & Proposed scheme & $\mathcal{I E}$ & - & $1 I+46.4 M$ & $1 I+103.2 M$ & $1 I+211.6 M$ \\
\hline & Method 10 & $\mathcal{I E}$ & - & $1 I+46.8$ & $1 I+102.0 M$ & $1 I+212.4 M$ \\
\hline & Proposed scheme & $\mathcal{J}$ & $1 I+10.2 M$ & $1 I+42.4 M$ & $1 I+93.4 M$ & $1 I+194.0 M$ \\
\hline & LM Method [6], 13 & $\mathcal{J}$ & - & $1 I+36.6 M$ & $1 I+79.0 M$ & $1 I+163.8 M$ \\
\hline
\end{tabular}

In Table 6, we compare the costs of the described schemes to that of the proposed scheme from Section 4.1 for different windows $w$. Costs for the latter method are derived from Table 4 and Appendix D. As it can be seen, the new approach outperforms every other method in cases 1 and 2 for both $\mathcal{I E}$ and $\mathcal{J} \mathcal{Q}$. Note that the advantage increases with the window size. For instance, if $1 I=30 M$, the cost reduction can be as high as $25 \%(w=6, \mathcal{J} \mathcal{Q})$. Nevertheless, in case 2 with $\mathcal{I E}$ coordinates, both the proposed and traditional methods offer comparable performance.

In the case of standard curves, the LM scheme still achieves the highest performance. Nevertheless, for case 1, the modified LM scheme (7) and the new approach achieve similar performance.

In settings where inversions are not so expensive (low $I / M$ ratios), it could be attractive the implementation of case 3 . In this case, Table 7 shows the performance of the traditional approach and the proposed method on a standard curve form. Also, the $I / M$ ratios for which the traditional, the proposed and the LM method achieve the lowest cost are shown at the bottom of the table. As it can be observed, the LM method offers the highest performance for a wide range of high $I / M$ ratios on a standard curve, whereas the proposed method is convenient for low/intermediate values $I / M$.

Table 7. Costs of different schemes in affine (case 3) and $I / M$ ranges for which each scheme achieves the lowest cost on a standard curve; $1 M=0.8 S$

\begin{tabular}{|l|c|c|c|}
\hline Method & $w=4$ & $w=5$ & $w=6$ \\
\hline \hline Proposed scheme, case 3 & $3 I+19.4 M$ & $6 I+34.2 M$ & $11 I+60.2 M$ \\
Traditional & $4 I+12.0 M$ & $8 I+23.2 M$ & $16 I+45.6 M$ \\
\hline \hline$I / M$ range (LM Method (6), 13) & $I>8.6 M$ & $I>9 M$ & $I>10.4 M$ \\
$I / M$ range (Proposed, case 3) & $7.4 M<I<8.6 M$ & $5.5 M<I<9 M$ & $2.9 M<I<10.4 M$ \\
$I / M$ range (Traditional) & $I<7.4 M$ & $I<5.5 M$ & $I<2.9 M$ \\
\hline
\end{tabular}


Let us now compare the performance of our scheme for cases 1 and 2, to determine the best scheme for each scenario. For this analysis, we should also consider the scalar multiplication cost since different point operations apply to different cases. Note that we only analyze the performance on Edwards and Jacobi quartic curves, as these are the settings where our method has been shown to attain the lowest costs (see Table 6).

Let us consider the standard $w \mathrm{NAF}$ method. In this case, the cost of a scalar multiplication is approximately

$$
\begin{aligned}
& {\left[n \mathrm{D}+\left(\frac{\left(2^{w-2}-1\right)(n-1)}{2^{w-2}(w+1)}\right) \mathrm{A}+\left(\frac{(n-1)}{2^{w-2}(w+1)}\right) \mathrm{mA}\right]+\text { Cost }_{\text {Proposed, case } 1},} \\
& {\left[n \mathrm{D}+\left(\frac{n-1}{w+1}\right) \mathrm{mA}\right]+\text { Cost }_{\text {Proposed, case } 2,}}
\end{aligned}
$$

for cases 1 and 2, respectively. Table 8 shows the performance of the scalar multiplication including the costs of the precomputation schemes proposed in this work, cases 1 and 2. As it can be seen, case 1 achieves the best performance for most common $I / M$ ratios if $n=160$ bits. For higher security levels ( $n=512$ bits), the difference between case 1 and case 2 reduces and, ultimately, the most effective approach would be determined by the particular $I / M$ ratio of a given implementation. However, as the window size grows, case 1 would be again largely preferred. Therefore, for applications where memory is not scarce, case 1 would achieve the lowest cost. Similar conclusions are observed for $\mathcal{I E}$ coordinates, whose costs are not included in Table 8 because of space constraints.

Finally, we analyze the performance of the proposed scheme for tables $c_{i} P \pm$ $d_{i} Q$. In this case, a multiple scalar multiplication using the $\mathrm{JSF}_{3}$ method [9] costs approximately $[n \mathrm{D}+0.3083(n-1) \mathrm{A}+0.0617(n-1) \mathrm{mA}]+$ Cost $_{\text {Proposed }}$ case 1 and $[n \mathrm{D}+0.37(n-1) \mathrm{mA}]+$ Cost $_{\text {Proposed, }}$ case 2 for cases 1 and 2 , respectively. The latter can be reduced in the case of $\mathcal{J}$ coordinates if we consider the efficient DA operation 13]. The costs in this case are expressed by $[(0.63 n+0.37) \mathrm{D}+$ $0.3083(n-1) \mathrm{DA}+0.0617(n-1) \mathrm{mDA}]+$ Cost $_{\text {Proposed }}$, case 1 and $[(0.63 n+$ $0.37) \mathrm{D}+0.37(n-1) \mathrm{mDA}]+$ Cost $_{\text {Proposed, }}$ case 2 .

Table 9 shows the performance of the scalar multiplication including the costs of our precomputation scheme, cases 1 and 2. Similarly to the case of single scalar multiplication (see Table 8 ), case 1 achieves the best performance for most common $I / M$ ratios for $n=160$ bits with $\mathcal{J} \mathcal{Q}$ and $\mathcal{I E}$ coordinates. However, if $n=512$ bits, the range of $I / M$ ratios for which case 2 is more efficient

Table 8. Cost of scalar multiplication using $w \mathrm{NAF}$ and the proposed scheme (cases 1 and 2); and $I / M$ range for which case 1 achieves the lowest cost on $\mathcal{J} \mathcal{Q}$ coord

\begin{tabular}{|l|c|c|c|c|c|}
\hline \multirow{2}{*}{ Method } & \multicolumn{2}{|c|}{$n=160$ bits } & \multicolumn{3}{|c|}{$n=512$ bits } \\
\cline { 2 - 6 } & $w=4$ & $w=5$ & $w=4$ & $w=5$ & $w=6$ \\
\hline \hline Proposed, case 1 & $1279.6 M$ & $1265.4 M$ & $4035.7 M$ & $3921.5 M$ & $3870.2 M$ \\
Proposed, case 2 & $1 I+1267.1 M$ & $1 I+1269.2 M$ & $1 I+3970.5 M$ & $1 I+3874.0 M$ & $1 I+3858.8 M$ \\
\hline \hline$I / M$ range (case 1) & $I>12.5 M$ & $I>0 M$ & $I>65.2 M$ & $I>47.5 M$ & $I>11.4 M$ \\
\hline
\end{tabular}


Table 9. Cost of multiple scalar multiplication using $\mathrm{JSF}_{3}$ and the proposed scheme (cases 1,2 ); and $I / M$ range in which case 1 achieves the lowest cost on $\mathcal{J}, \mathcal{I E}$ and $\mathcal{J} \mathcal{Q}$

\begin{tabular}{|l|c|c|c|c|c|c|}
\hline \multirow{2}{*}{ Method } & \multicolumn{3}{|c|}{$n=160$ bits } & \multicolumn{3}{c|}{$n=512$ bits } \\
\cline { 2 - 7 } & $\mathcal{J Q}$ & $\mathcal{I E}$ & $\mathcal{J}$ & $\mathcal{J \mathcal { Q }}$ & $\mathcal{I \mathcal { E }}$ & $\mathcal{J}$ \\
\hline \hline Proposed, case 1 & $1572.2 M$ & $1624.9 M$ & $1889.6 M$ & $4886.7 M$ & $5062.0 M$ & $5840.2 M$ \\
Proposed, case 2 & $1 I+1565.4 M$ & $1 I+1635.9 M$ & $1 I+1796.8 M$ & $1 I+4771.4 M$ & $1 I+4964.4 M$ & $1 I+5511.1 M$ \\
\hline \hline$I / M$ range (case 1) & $I>6.8 M$ & $I>0 M$ & $I>92.8 M$ & $I>115.3 M$ & $I>97.6 M$ & $I>329.1 M$ \\
\hline
\end{tabular}

increases significantly. Also, note that case 2 appears to be the best choice for $\mathcal{J}$ coordinates for a wide range of $I / M$ ratios.

As reference, the costs for $\mathcal{J} \mathcal{Q}$ and $\mathcal{J}$ using a traditional chain for precomputation are $1598 M$ or $1 I+1594 M$, and $1922 M$ or $1 I+1849 M$, respect. $(n=160)$.

\section{Other Applications}

We have discussed the application of the strategy of the conjugate addition to build efficient precomputation tables with the forms $d_{i} P$ and $c_{i} P \pm d_{i} Q$. However, this technique can be easily applied to other table forms such as the one required by the generalized JSF [19], which requires the computation of $\left(3^{k}-1\right) / 2-k$ non-trivial points. For instance, for $k=3$ scalars, the previous algorithm requires the precomputation of $P \pm Q, P \pm R, Q \pm R, P+Q \pm R, P-Q \pm R$, which costs about 10 general additions. With our strategy, the latter is reduced to only 5 addition/conjugate addition pairs (case 1 ). Note that the advantage grows exponentially with the number of scalars.

Other obvious application is the extension of our strategy to other settings such as binary fields. Let us illustrate the latter with the addition formula due to [14] and later refined by [5]. The cost of adding two points $P+Q$ with the latter formula takes $13 M+4 S$. Then, if we need the value $P-Q$ right after, we can store most partial results from the original addition and obtain the previous value with a cost of only $5 M$ by noticing that $-Q=\left(X_{2}, X_{2} Z_{2}+Y_{2}, Z_{2}\right)$ in Lopez-Dahab coordinates. Note that the partial term $Y_{2} Z_{1}^{2}$ from the original formula is replaced by $-Y_{2} Z_{1}^{2}=\left(X_{2} Z_{2}+Y_{2}\right) Z_{1}^{2}=X_{2} Z_{2} Z_{1}^{2}+Y_{2} Z_{1}^{2}$, which only cost one extra multiplication. Straightforward generalizations of this technique (and also of the proposed precomputation schemes) can be applied to other coordinate systems and/or elliptic curve forms.

\section{Conclusions}

We have introduced an innovative technique based on conjugate additions that can be efficiently exploited to reduce costs in a scalar multiplication. The relevant formulas on three different settings (namely, standard, Jacobi quartic and Edwards curves) over prime fields have been derived and shown to attain significant cost reductions in comparison with traditional formulae. In particular, 
we have proposed novel precomputation schemes based on this technique. Our analysis shows that the new schemes are especially attractive on the highly efficient Jacobi quartic and Edwards curves, enabling even faster implementations. Finally, we have also discussed other applications of the introduced strategy to binary fields and other precomputation tables.

Acknowledgments. We would like to thank the Natural Sciences and Engineering Research Council of Canada (NSERC) and the Ontario Centres of Excellence (OCE) for partially supporting this work. Also, after finishing this work, we became aware of a similar idea proposed by M. Scott during a presentation in ECC2008. He rediscovered the idea of exploiting similarities between $P+Q$ and $P-Q$ and suggested a slightly different sequence to precompute points.

\section{References}

1. Bernstein, D., Lange, T.: Faster Addition and Doubling on Elliptic Curves. In: Kurosawa, K. (ed.) ASIACRYPT 2007. LNCS, vol. 4833, pp. 29-50. Springer, Heidelberg (2007)

2. Bernstein, D., Lange, T.: Inverted Edwards Coordinates. In: Boztas, S., Lu, H.F(F.) (eds.) AAECC 2007. LNCS, vol. 4851, pp. 20-27. Springer, Heidelberg (2007)

3. Brown, M., Hankerson, D., Lopez, J., Menezes, A.: Software Implementation of the NIST Elliptic Curves over Prime Fields. In: Naccache, D. (ed.) CT-RSA 2001. LNCS, vol. 2020, pp. 250-265. Springer, Heidelberg (2001)

4. FIPS PUB 186-2: Digital Signature Standard (DSS). National Institute of Standards and Technology (NIST) (2000)

5. Higuchi, A., Takagi, N.: A Fast Addition Algorithm for Elliptic Curve Arithmetic in $\operatorname{GF}\left(2^{n}\right)$ using Projective Coordinates. Information Processing Letters 76(3), 101-103 (2000)

6. Hisil, H., Wong, K., Carter, G., Dawson, E.: Faster Group Operations on Elliptic Curves. Cryptology ePrint Archive, Report 2007/441 (2007)

7. Hisil, H., Wong, K., Carter, G., Dawson, E.: An Intersection Form for JacobiQuartic Curves. Personal communication (2008)

8. Koblitz, N.: Elliptic Curve Cryptosystems. Mathematics of Computation, vol. 48, pp. 203-209 (1987)

9. Kuang, B., Zhu, Y., Zhang, Y.: An Improved Algorithm for uP+vQ using $\mathrm{JSF}_{3}$. In: Jakobsson, M., Yung, M., Zhou, J. (eds.) ACNS 2004. LNCS, vol. 3089, pp. 467-478. Springer, Heidelberg (2004)

10. Lim, C.H., Hwang, H.S.: Fast Implementation of Elliptic Curve Arithmetic in GF $\left(p^{n}\right)$. In: Imai, H., Zheng, Y. (eds.) PKC 2000. LNCS, vol. 1751, pp. 405-421. Springer, Heidelberg (2000)

11. Longa, P.: ECC Point Arithmetic Formulae (EPAF), http://patricklonga.bravehost.com/jacobian.html

12. Longa, P., Miri, A.: Fast and Flexible Elliptic Curve Point Arithmetic over Prime Fields. IEEE Trans. Comp. 57(3), 289-302 (2008)

13. Longa, P., Miri, A.: New Composite Operations and Precomputation Scheme for Elliptic Curve Cryptosystems over Prime Fields. In: Cramer, R. (ed.) PKC 2008. LNCS, vol. 4939, pp. 229-247. Springer, Heidelberg (2008) 
14. López, J., Dahab, R.: Improved Algorithms for Elliptic Curve Arithmetic in GF $\left(2^{\mathrm{n}}\right)$. Technical Report IC-98-39, Relatorio Técnico (1998)

15. Meloni, N.: New Point Addition Formulae for ECC Applications. In: Carlet, C., Sunar, B. (eds.) WAIFI 2007. LNCS, vol. 4547, pp. 189-201. Springer, Heidelberg (2007)

16. Miller, V.: Use of Elliptic Curves in Cryptography. In: Williams, H.C. (ed.) CRYPTO 1985. LNCS, vol. 218, pp. 417-426. Springer, Heidelberg (1986)

17. Möller, B.: Algorithms for Multi-exponentiation. In: Vaudenay, S., Youssef, A.M. (eds.) SAC 2001. LNCS, vol. 2259, pp. 165-180. Springer, Heidelberg (2001)

18. Okeya, K., Takagi, T., Vuillaume, C.: Efficient Representations on Koblitz Curves with Resistance to Side Channel Attacks. In: Boyd, C., González Nieto, J.M. (eds.) ACISP 2005. LNCS, vol. 3574, pp. 218-229. Springer, Heidelberg (2005)

19. Proos, J.: Joint Sparse Forms and Generating Zero Columns when Combing. Technical Report CORR 2003-23, University of Waterloo (2003)

20. Solinas, J.: Low-Weight Binary Representations for Pairs of Integers. Technical Report CORR 2001-41, University of Waterloo (2001)

\section{A Conjugate (Mixed) Addition in Jacobian Coordinates}

In the case of general addition, refer to equations (11) and (2) in Section 3.

In the case of mixed addition, let $P=\left(X_{1}, Y_{1}, Z_{1}\right)$ and $Q=\left(x_{2}, y_{2}\right)$ be two points on an elliptic curve $E$. If the mixed addition is performed using [12, formula (16)] and the partial values $\left(4 \beta^{3}+8 X_{1} \beta^{2}\right), 4 X_{1} \beta^{2},-8 Y_{1} \beta^{3}, Z_{3}$ and $Z_{1}^{3} y_{2}$ are temporarily stored, the conjugate mixed addition $P-Q=P+(-Q)=$ $\left(X_{1}, Y_{1}, Z_{1}\right)+\left(x_{2},-y_{2}\right)=\left(X_{4}, Y_{4}, Z_{4}\right)$ can be performed as follows:

$$
X_{4}=\gamma^{2}-\left(4 \beta^{3}+8 X_{1} \beta^{2}\right), Y_{4}=\gamma\left(4 X_{1} \beta^{2}-X_{4}\right)-8 Y_{1} \beta^{3}, Z_{4}=Z_{3}
$$

where $\gamma=-2\left(Z_{1}^{3} y_{2}+Y_{1}\right)$. This formula only costs $1 M+1 S+4 A+1(\times 2)$.

\section{B Conjugate (Mixed) Addition in $\mathcal{J} \mathcal{Q}$ Coordinates}

Let $P=\left(X_{1}, Y_{1}, Z_{1}, X_{1}^{2}, Z_{1}^{2}\right)$ and $Q=\left(X_{2}, Y_{2}, Z_{2}, X_{1}^{2}, Z_{1}^{2}\right)$ be two points on a Jacobi quartic curve. If the addition $P+Q$ is performed using the following formula due to [6]:

$$
\begin{gathered}
X_{3}=\left(\alpha+2 Y_{1}\right)\left(\beta+2 Y_{2}\right)-\alpha \beta-4 Y_{1} Y_{2}, Z_{3}=\phi-\theta \\
Y_{3}=(\theta+\phi+2 \alpha \beta)\left[4\left(X_{1}^{2}+Z_{1}^{2}\right)\left(X_{2}^{2}+Z_{2}^{2}\right)+a \alpha \beta+4 Y_{1} Y_{2}\right]-16\left(X_{3}^{2}+Z_{3}^{2}\right), \\
X_{3}^{2}=\left(X_{3}\right)^{2}, Z_{3}^{2}=\left(Z_{3}\right)^{2},
\end{gathered}
$$

where $\phi=4 Z_{1}^{2} Z_{2}^{2}, \theta=4 X_{1}^{2} X_{2}^{2}, \alpha=\left(X_{1}+Z_{1}\right)^{2}-\left(X_{1}^{2}+Z_{1}^{2}\right), \beta=\left(X_{2}+Z_{2}\right)^{2}-$ $\left(X_{2}^{2}+Z_{2}^{2}\right)$, and the partial values $\beta,\left(\alpha+2 Y_{1}\right), 2 Y_{2}, \alpha \beta,-4 Y_{1} Y_{2},\left(4 X_{1}^{2} X_{2}^{2}+\right.$ $\left.4 Z_{1}^{2} Z_{2}^{2}\right), 2 \alpha \beta, 4\left(X_{1}^{2}+Z_{1}^{2}\right)\left(X_{2}^{2}+Z_{2}^{2}\right)+4 Y_{1} Y_{2}, a \alpha \beta, Z_{3}$ and $Z_{3}^{2}$ are temporarily stored, then the conjugate addition $P-Q=P+(-Q)=\left(X_{1}, Y_{1}, Z_{1}, X_{1}^{2}, Z_{1}^{2}\right)+$ $\left(-X_{2}, Y_{2}, Z_{2}, X_{2}^{2}, Z_{2}^{2}\right)=\left(X_{4}, Y_{4}, Z_{4}\right)$ can be performed with only $2 M+1 S+$ $7 A+1(\times 16)$ as follows: 


$$
\begin{gathered}
X_{4}=\left(\alpha+2 Y_{1}\right)\left(-\beta+2 Y_{2}\right)+\alpha \beta-4 Y_{1} Y_{2}, Z_{4}=\phi-\theta=Z_{3}, \\
Y_{4}=(\theta+\phi-2 \alpha \beta)\left[4\left(X_{1}^{2}+Z_{1}^{2}\right)\left(X_{2}^{2}+Z_{2}^{2}\right)-a \alpha \beta+4 Y_{1} Y_{2}\right]-16\left(X_{4}^{2}+Z_{4}^{2}\right), \\
X_{4}^{2}=\left(X_{4}\right)^{2}, Z_{4}^{2}=Z_{3}^{2},
\end{gathered}
$$

In the case of mixed addition, let $P=\left(X_{1}, Y_{1}, Z_{1}, X_{1}^{2}, Z_{1}^{2}\right)$ and $Q=\left(x_{2}, y_{2}, x_{2}^{2}\right)$ be two points on a Jacobi quartic curve. If the mixed addition $P+Q$ is performed using the following formula due to [6]:

$$
\begin{gathered}
X_{3}=\left(\alpha+2 Y_{1}\right)\left(x_{2}+y_{2}\right)-\alpha x_{2}-2 Y_{1} y_{2}, Z_{3}=2\left(Z_{1}^{2}-X_{1}^{2} x_{2}^{2}\right), \\
Y_{3}=2\left(\left(X_{1}^{2} x_{2}^{2}+Z_{1}^{2}+\alpha x_{2}\right)\left[2\left(X_{1}^{2}+Z_{1}^{2}\right)\left(x_{2}^{2}+1\right)+a \alpha x_{2}+2 Y_{1} y_{2}\right]-2\left(X_{3}^{2}+Z_{3}^{2}\right)\right), \\
X_{3}^{2}=\left(X_{3}\right)^{2}, Z_{3}^{2}=\left(Z_{3}\right)^{2},
\end{gathered}
$$

where $\alpha=\left(X_{1}+Z_{1}\right)^{2}-\left(X_{1}^{2}+Z_{1}^{2}\right)$, and the partial values $\left(\alpha+2 Y_{1}\right), \alpha x_{2}$, $-2 Y_{1} y_{2},\left(X_{1}^{2} x_{2}^{2}+Z_{1}^{2}\right),\left(2\left(X_{1}^{2}+Z_{1}^{2}\right)\left(x_{2}^{2}+1\right)+2 Y_{1} y_{2}\right), a \alpha x_{2}, Z_{3}$ and $Z_{3}^{2}$ are temporarily stored, then the conjugate mixed addition $P-Q=P+(-Q)=$ $\left(X_{1}, Y_{1}, Z_{1}, X_{1}^{2}, Z_{1}^{2}\right)+\left(-x_{2}, y_{2}, x_{2}^{2}\right)=\left(X_{4}, Y_{4}, Z_{4}\right)$ can be performed with $2 M+$ $1 S+7 A+2(\times 2)$ as follows:

$$
\begin{gathered}
X_{4}=\left(\alpha+2 Y_{1}\right)\left(-x_{2}+y_{2}\right)+\alpha x_{2}-2 Y_{1} y_{2}, Z_{4}=2\left(Z_{1}^{2}-X_{1}^{2} x_{2}^{2}\right)=Z_{3}, \\
Y_{4}=2\left(\left(X_{1}^{2} x_{2}^{2}+Z_{1}^{2}-\alpha x_{2}\right)\left[2\left(X_{1}^{2}+Z_{1}^{2}\right)\left(x_{2}^{2}+1\right)-a \alpha x_{2}+2 Y_{1} y_{2}\right]-2\left(X_{4}^{2}+Z_{4}^{2}\right)\right), \\
X_{4}^{2}=\left(X_{4}\right)^{2}, Z_{4}^{2}=Z_{3}^{2} .
\end{gathered}
$$

\section{Conjugate (Mixed) Addition in $\mathcal{I E}$ Coordinates}

Let $P=\left(X_{1}, Y_{1}, Z_{1}\right)$ and $Q=\left(X_{2}, Y_{2}, Z_{2}\right)$ be two points on Inverted Edwards coordinates. If the general addition $P+Q$ is performed using the following formula due to [2] (note that some terms have been rearranged to save a few field additions):

$$
\begin{gathered}
X_{3}=\left[\alpha+d\left(Z_{1} Z_{2}\right)^{2}\right]\left(X_{1} X_{2}-Y_{1} Y_{2}\right), Y_{3}=\left[\alpha-d\left(Z_{1} Z_{2}\right)^{2}\right]\left(X_{1} Y_{2}+X_{2} Y_{1}\right) \\
Z_{3}=Z_{1} Z_{2}\left(X_{1} X_{2}-Y_{1} Y_{2}\right)\left(X_{1} Y_{2}+X_{2} Y_{1}\right)
\end{gathered}
$$

where $\alpha=X_{1} X_{2} Y_{1} Y_{2}$, and the partial values $\left[X_{1} X_{2} Y_{1} Y_{2}+d\left(Z_{1} Z_{2}\right)^{2}\right], X_{1} X_{2}$, $Y_{1} Y_{2},\left[X_{1} X_{2} Y_{1} Y_{2}-d\left(Z_{1} Z_{2}\right)^{2}\right], X_{1} Y_{2}, X_{2} Y_{1}$ and $Z_{1} Z_{2}$ are temporarily stored, then the conjugate addition $P-Q=P+(-Q)=\left(X_{1}, Y_{1}, Z_{1}\right)+\left(-X_{2}, Y_{2}, Z_{2}\right)=$ $\left(X_{4}, Y_{4}, Z_{4}\right)$ can be performed with the following (with a cost of only $\left.4 M+2 A\right)$ :

$$
\begin{gathered}
X_{4}=\left[\alpha-d\left(Z_{1} Z_{2}\right)^{2}\right]\left(X_{1} X_{2}+Y_{1} Y_{2}\right), Y_{4}=-\left[\alpha+d\left(Z_{1} Z_{2}\right)^{2}\right]\left(X_{1} Y_{2}-X_{2} Y_{1}\right), \\
Z_{4}=-Z_{1} Z_{2}\left(X_{1} X_{2}+Y_{1} Y_{2}\right)\left(X_{1} Y_{2}-X_{2} Y_{1}\right),
\end{gathered}
$$

The formula for mixed addition can be obtained by setting $Z_{2}=1$ in formula (17) and has a cost of $9 M+1 S+4 A$. Then, if the partial values $\left(X_{1} x_{2} Y_{1} y_{2}+d Z_{1}^{2}\right)$, $X_{1} x_{2}, Y_{1} y_{2},\left(X_{1} x_{2} Y_{1} y_{2}-d Z_{1}^{2}\right), X_{1} y_{2}$ and $x_{2} Y_{1}$ are temporarily cached, then the conjugate mixed addition $P-Q=P+(-Q)=\left(X_{1}, Y_{1}, Z_{1}\right)+\left(-x_{2}, y_{2}\right)=$ $\left(X_{4}, Y_{4}, Z_{4}\right)$ can be performed by: 


$$
\begin{gathered}
X_{4}=\left[X_{1} x_{2} Y_{1} y_{2}-d Z_{1}^{2}\right]\left(X_{1} x_{2}+Y_{1} y_{2}\right), Y_{4}=-\left[X_{1} x_{2} Y_{1} y_{2}+d Z_{1}^{2}\right]\left(X_{1} y_{2}-x_{2} Y_{1}\right), \\
Z_{4}=-Z_{1}\left(X_{1} x_{2}+Y_{1} y_{2}\right)\left(X_{1} y_{2}-x_{2} Y_{1}\right)
\end{gathered}
$$

which only costs $4 M+2 A$. We remark that memory requirements of the new conjugate formulas can be minimized by performing $P+Q$ and $P-Q$ in an "interlaced" fashion (see, for instance, Table 2).

\section{Calculation of Precomputed Points}

The table below shows the proposed precomputing sequences for various values $m$. For $m=5$ the first sequence corresponds to $\mathcal{J}$ and $\mathcal{J} \mathcal{Q}$, and the second one to $\mathcal{I E}$. Tied arrows denote addition/conjugate addition pairs (or mixed addition/conjugate mixed addition pairs if performed with affine point $P$ ).

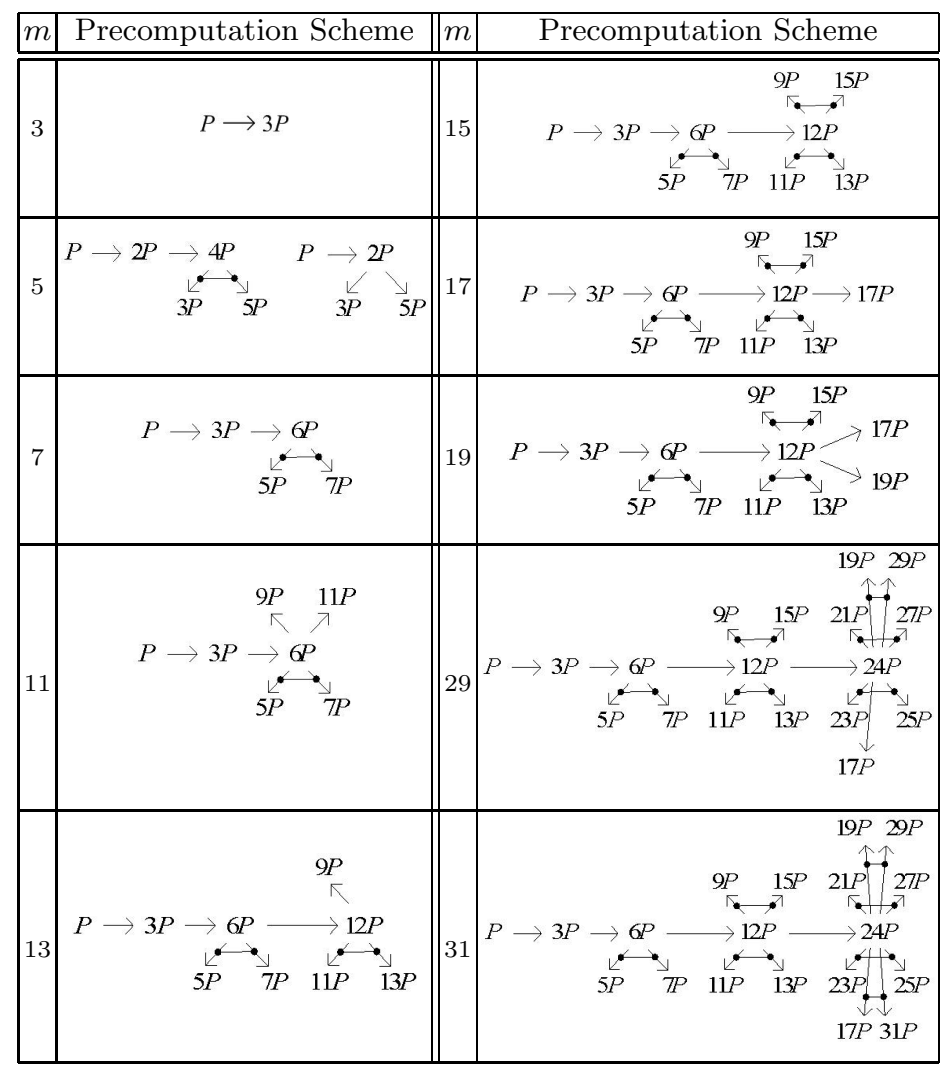

